\title{
The Effect of Green logistics on Economic growth, Social and Environmental sustainability: An Empirical study of Developing countries in Asia
}

\author{
Prof. Syed Abdul Rehman Khan \\ School of Economics and Management, Tsinghua University, Beijing, China \\ Khan syed@sem.tsinghua.edu.cn
}

\begin{abstract}
This panel study investigates the relationship between green logistics indices, economic, environmental, and social factors in the perspective of Asian emerging economies. This study adopted FMOLS and DOLS methods to test research hypothesis, catering the problem of endogenity and serial correlation. The results suggest that logistics operations, particularly LPI2 (efficiency of customs clearance processes), LPI4 (quality of logistics services) and LPI5 (trade and transportrelated infrastructure), are positively and significantly correlated with per capita income, manufacturing value added and trade openness. While, greater logistics operations are negatively associated with social and environmental problems including, climate change, global warming, carbon emissions, and poisoning atmosphere. In addition, human health is badly affected by heavy smog, acid rainfall, and water pollution. The findings further extend and reveal that political instability, natural disaster and terrorism are also a primary cause of poor economic growth and environmental sustainability with poor trade and logistics infrastructure. Further, the application of renewable energy resources and green practices can mitigate negative effects on social and environmental sustainability without compromising the performance of economic growth. There is very limited empirical work presented in literature using renewable energy and green ideology to solve macro-level social and environmental problems, while this study will assist the policymakers and researchers to understand the importance of green concept in improving countries' social, economic and environmental performance.
\end{abstract}

Keywords: Renewable energy; Education expenditure; Environmental degradation; Health expenditure; Carbon emissions; and foreign direct investments

\section{Introduction}

In $21^{\text {st }}$ century, logistics plays a vital role in connecting cities, countries and continents around the globe. It has a function of integration in global supply chain management, which means, logistics contains a complete set of integrated activities including, freight transport, information sharing and inventory storage with members of supply chain (Khan et al. 2018). In recent years, logistics industry has attracted much attention in scholarly literature due to its advantages and disadvantages on countries' economic growth and environment performance respectively (Aldakhil et al. 2018; Khan and Dong, 2017a; Martel and Klibi. 2016). A number of firms have started to adopte ecological practices in their logistics operations to achieve economic and social 
advantages without compromising environmental sustainability, while in developing countries of Asia, most times green practices in supply chain and logistics have insignificant and/or negative effect on firms' profitability e.g., Khan and Dong, (2017b) conducted a research to investigate the impact of green supply chain on manufacturing firms' economic and environmental performance in developing countries of Asia particularly Pakistan. They found that green practices including ecological design of products, cooperation with customers, green purchasing of raw materials and components, green transportation and distribution have significantl positive relationship with environmental performance. However, green practices, especially green purchasing, have negative effects on a firm's profitability due to heavy taxes and import duties on green material and components, while most green materials are imported from European and Western world, incurring extra cost for the system under the name of long lead-time and insurance cost (Khan et al., 2016a).

According to the report of WHO, (2017) more than 2.1 million people die around the globe from air pollution on a yearly basis, while Asian region is the most polluted area in the world and its PM 2.5 (Particulate matter) has increased till alarming situation, particularly in emerging economies such as China, India, Bangladesh and Pakistan. In addition, WHO, (2017) the report also indicated that Air pollution in some Asian cities including Beijing, Xi'an, Delhi, Bombay, Dhaka, Karachi, Lahore and Islamabad is so bad that at times, the cities were enveloped by a blanket of smog that impedes visibility. The IEA (International Energy Agency) highlighted some basic causes of air pollution including energy, manufacturing and transportation sector, which contribute to carbon emissions by $42 \%, 19 \%$ and $23 \%$ respectively. Unquestionably, global logistics operations heavily depend on energy, especially fossil fuel, resulting in environmental deterioration and grave repercussion on public health.

The concept of green logistics is developed to mitigate harmful effects of logistics on social and environmental sustainability without compromising profitability and efficiency of logistics operations The report of EU (European Union) identified that more than 10.3 million workforce were associated with logistics and transport sectors, covering $4.5 \%$ of total employment around the globe (EUROPA, 2011). Logistics industry mainly rely on fossil fuel and this industry consumes approximately $96 \%$ of its energy needs. For that reason, logistics and transport sectors have greater contribution to carbon emissions, PM 2.5 (fine particles), nitrogen and greenhouse gas emissions (EUROPA, 2017).

Zaman \& Shamsuddin, (2017) carried out a research on European countries to investigate the relationship between logistics and energy demand. The results showed that logistics operations have strong correlation with energy demand. Logistics industry plays a significant role in economic development of countries but countries need to pay price in terms of poor environmental performance. Khan et al., (2017a) argued that application of renewable energy and green practices in logistics operations are the only solution to control air pollution, climate change and global warming problems. Aldakhil et al., (2018) conducted a panel study on BRICS countries to develop an integrated model for green business. They found that through adoption of renewable energy and 
green practices in logistics operations, most of BRICS countries, such as South African, Brazil and Russia, their environmental performance has improved without any negative influence and/or slowdown on economic growth, while India and China are still suffering from heavy smog, PM 2.5 and carbon emissions, which not only damage their reputation and image on international forum but also seriously affect their people's health in terms of different pollution intensive diseases e.g., asthma, lungs cancer, and Cardiovascular Diseases.

\section{Research Motivation and Objective}

WHO (2017) highlights that Asian developing countries were suffering from serious social and environmental problems including health diseases, carbon emissions, and climate change. Since last couple of decades, Asian developing countries have been mainly affected by terrorist attacks and natural disasters, dropping billions of dollars from economy and damaging logistics and transport-related infrastructure like airports, seaports and national highways. The global terrorism reports showed that only in last couple of years, Afghanistan, Pakistan and India suffered 7,600, 6,664, and 4,407 terrorist attacks respectively. While, this study is to investigate the relationship between countries' logistics operational performance and national scale social, economic and environmental indicators in a panel of Asian emerging economies during the period of 2001 to 2017. The rest of study is organized as followed: section 2 presents literature review and formulation of hypothesis to achieve the goal of this study. Section 3 covers source of data and research methodology. Section 4 is based on research findings and discussion. Finally, section 5 contains concluding remarks, implication of policy, research limitations and future research work.

\section{Literature review}

\subsection{Relationship between Green logistics, Economic and Environmental Sustainability}

Logistics sector plays a significant role in economic development, while it also brings badness to environmental sustainability in the long run (Petrini and Pozzebon 2009). A panel study was conducted by Aldakhil et al., (2018) to investigate the determinants of green logistics in BRICS member states. The findings show that green practices in logistics operations is positively correlated with sustainable economic and environmental growth. Lai et al., (2004) did a cross-sectional survey for three transport logistics sectors, namely, air and water transport, freight forwarding and third party logistics. They found that all these sectors have a sustained impact on supply chain performance and logistics. Khan et al., (2018) conducted an empirical research on developed countries in Europe in order to explore the impact of logistics performance on macro-economic and environmental indicators. The results show that greater performance of logistics spurs country's economic growth but has a negative effect on environmental sustainability in terms of air pollution, climate change and global 
warming. Khan and Dong, (2017a) warned that government and regulatory authorities should bring strict environmental policies into force as soon as possible and also encourage renewable/green energy as an alternative of fossil fuel in manufacturing and logistics industry for better environmental sustainability. Otherwise, it will be very late to take actions to protect environment and will be impossible to restore natural resources.

The adoption of green practices in logistics and supply chain operations emphasizes the waste reduction for better environmental performance, while green practices directly leads towards cost reduction, improving the efficiency of operations and performance (Jr et al., 2012; Ruamsook et al., 2009). Nikolaou et al., (2013) introduced an integrated model for CSR (corporate social responsibility) based on micro-economic, social and environmental indicators under the presence of reverse logistics system conducted that for overall sustainability and resolving financial matters. The results indicate that the system of reverse logistics plays a significant role in strengthening the GMP (green management practices) in an organizational settings. Khan and Dong (2017b) examined the impacts of green practices in logistics and supply chain operations on firms' environmental and economic performance. In their research, the data were collected from manufacturing companies and linear regression method was employed. The results indicate that green practices improve the economic performance in terms of greater level of customer satisfaction and trust, increase the efficiency of operations, and enhance environmental sustainability such as reduction in carbon emissions and solid waste. In similar line Acquaye et al., (2017and 2014); Khan et al., (2016b); Sarkis et al. (2010) and Van Hoek, (1999) confirmed achieving economic and environmental sustainability targets depends on the viability and efficiency of green practices in logistics operations.

Chaudhry et al., (2018) highlighted that when continuous terrorist attacks happened in Asian developing countries, particularly in Afghanistan, India and Pakistan, their economic growth declined. In addition, logistics and transport-related infrastructure also has been damaged due to bombing attacks and armed assault, creating direct negative effects on trade. These countries are portrayed as terrorist states on global forum. Khan et al., (2017a) investigate the cause of handsome economic growth in European countries with macro-economic data. The results indicate that European countries have modern and improved logistics and transport-related infrastructure, backing the economic growth and attracting foreign direct investment inflows. Rodrigue (2017) the logistics and transport industry releases millions tons of gases into the atmosphere on yearly basis, 25 to $30 \%$ of greenhouse emissions, which is a serious cause of human diseases and environmental degradation. In addition, as a contributor to climate change, transportation is also influenced, particularly over infrastructure (e.g. more floods due to rising sea levels) and operations (harsher operating conditions) (Bektas et al., 2016). The above cited studies highlighted the importance of green practices in logistics and supply chain operations; for that reason, it is desirable to mitigate environmental concerns and enhanced economic growth through implementation of green ideology in logistics operations. The study hypothesis that: 
H1: Improved green logistics performance spur economic growth of countries

H2: Greater environmental concerns in logistics operations are positively associated with the enhanced performance of green logistics

\subsection{The relationship between green practices in logistics operations and macro- level social factors}

Burrell, (2006) highlighted that most manufacturing and logistics operations have harmful effects on our society, resulting in environmental degradation and human diseases including ischaemic heart disease, lung cancer, stroke, cholera, Amoebiasis, giardiasis and hepatitis (Acquaye et al., 2015; Khan et al., 2018). For decades, companies have been mainly emphasizing on corporate social responsibility so as to significantly mitigate their logistics and manufacturing operations' negative effects on society (Rosdríguez et al., 2014). A study conducted by Visser, (2008) explains the responsibility of corporate sectors is to engage in education and training programs, free healthcare, influencing political reforms, and creating awareness to obey governmental regulations including protection of natural resources for better environmental sustainability.

In some developing countries of Asia, corporate sector undertakes more social responsibilities in different sectors particularly in healthcare and education due to absence of social welfare from governmental bodies (Rodríguez et al., 2014). Due to industrialization, air, water and solid pollution have been increasing, which is primary cause of climate change and global warming, while the whole society is suffering from different diseases (Sense \& Governance, 2011). Aldakhil et al. (2018) highlighted that global logistics operations are a main contributor to carbon emissions and greenhouse gases in atmosphere, while it can be mitigated through implementing green practices in logistics operations. Blind, (2001) and Khan et al., (2016c) emphasized that regulatory authorities and corporate sector should provide basic environmental friendly education to people, increasing the importance of natural resources in their mind so that people start to buy ecological products by themselves. In addition, ecological behaviors of customers will create pressure on firms to adopt green practices in their manufacturing and logistics operations. (Zaman and Shamsuddin, 2017; Khan and Dong, 2017a). Khan et al., (2018) identified that green logistics operations and manufacturing practices improve air and water quality due to reduction in carbon emissions and solid waste, which also have positive effects on the whole society. On the basis of above cited studies, we have developed the following hypothesis.

\section{H3: Polluted practices in supply chain and logistics operation are positively correlated with social problems}

\section{Methodology}

This research explores the relationship among green logistics and national scale economic, social and environmental factors in a panel of Asian emerging economies during the period of 2001 to 2017. Undeniably, logistics operations play a significant 
role in economic development, which is called backbone of economy. In the absence of green practices in supply chain and environmental friendly policies, however, logistics operations are also a basic cause of environmental and social problems. Hence, this empirical study integrates green logistics operations with social, environmental factors and economic growth under green business practices and policies. The following equations show the green logistics determinants in a panel of Asian emerging economies i.e.,

$$
\begin{gathered}
\ln (L P I 1)_{i, t}=a_{0}+a_{1} \ln (F D I)_{i, t}+a_{2} \ln (G D P P C)_{i, t}+a_{3} \ln (T O P)_{i, t}+ \\
a_{4} \ln (I N D V)_{i, t}+a_{5} \ln (M V D)_{i, t}+a_{6} \ln (F O S S I L)_{i, t}+a_{7} \ln (T G H G)_{i, t}+ \\
a_{8} \ln (N O X)_{i, t}+a_{9} \ln (C O 2)_{i, t}+a_{10} \ln (R E C)_{i, t}+a_{11} \ln (\mathrm{HXP})_{i, t}+ \\
a_{12} \ln (\text { CRIME })_{i, t}+a_{13} \ln (E D U)_{i, t}+a_{14} \ln (P O L I)_{i, t}+\epsilon_{i, t}
\end{gathered}
$$

$$
\begin{gathered}
\ln (L P I 2)_{i, t}=a_{0}+a_{1} \ln (F D I)_{i, t}+a_{2} \ln (G D P P C)_{i, t}+a_{3} \ln (T O P)_{i, t}+ \\
a_{4} \ln (I N D V)_{i, t}+a_{5} \ln (M V D)_{i, t}+a_{6} \ln (\text { FOSSIL })_{i, t}+a_{7} \ln (T G H G)_{i, t}+ \\
a_{8} \ln (N O X)_{i, t}+a_{9} \ln (C O 2)_{i, t}+a_{10} \ln (R E C)_{i, t}+a_{11} \ln (\mathrm{HXP})_{i, t}+ \\
a_{12} \ln (C R I M E)_{i, t}+a_{13} \ln (E D U)_{i, t}+a_{14} \ln (P O L I)_{i, t}+\epsilon_{i, t} \text { (2) } \\
\ln (L P I 3)_{i, t}=a_{0}+a_{1} \ln (F D I)_{i, t}+a_{2} \ln (G D P P C)_{i, t}+a_{3} \ln (T O P)_{i, t}+ \\
a_{4} \ln (I N D V)_{i, t}+a_{5} \ln (M V D)_{i, t}+a_{6} \ln (F O S S I L)_{i, t}+a_{7} \ln (T G H G)_{i, t}+ \\
a_{8} \ln (N O X)_{i, t}+a_{9} \ln (C O 2)_{i, t}+a_{10} \ln (R E C)_{i, t}+a_{11} \ln (\mathrm{HXP})_{i, t}+ \\
a_{12} \ln (C R I M E)_{i, t}+a_{13} \ln (E D U)_{i, t}+a_{14} \ln (P O L I)_{i, t}+\epsilon_{i, t}
\end{gathered}
$$

$$
\begin{gathered}
\ln (L P I 4)_{i, t}=a_{0}+a_{1} \ln (F D I)_{i, t}+a_{2} \ln (G D P P C)_{i, t}+a_{3} \ln (T O P)_{i, t}+ \\
a_{4} \ln (I N D V)_{i, t}+a_{5} \ln (M V D)_{i, t}+a_{6} \ln (F O S S I L)_{i, t}+a_{7} \ln (T G H G)_{i, t}+ \\
a_{8} \ln (N O X)_{i, t}+a_{9} \ln (C O 2)_{i, t}+a_{10} \ln (R E C)_{i, t}+a_{11} \ln (\mathrm{HXP})_{i, t}+ \\
a_{12} \ln (C R I M E)_{i, t}+a_{13} \ln (E D U)_{i, t}+a_{14} \ln (P O L I)_{i, t}+\epsilon_{i, t}
\end{gathered}
$$

$$
\begin{gathered}
\ln (L P I 5)_{i, t}=a_{0}+a_{1} \ln (F D I)_{i, t}+a_{2} \ln (G D P P C)_{i, t}+a_{3} \ln (T O P)_{i, t}+ \\
a_{4} \ln (I N D V)_{i, t}+a_{5} \ln (M V D)_{i, t}+a_{6} \ln (F O S S I L)_{i, t}+a_{7} \ln (T G H G)_{i, t}+ \\
a_{8} \ln (N O X)_{i, t}+a_{9} \ln (C O 2)_{i, t}+a_{10} \ln (R E C)_{i, t}+a_{11} \ln (\mathrm{HXP})_{i, t}+ \\
a_{12} \ln (\text { CRIME })_{i, t}+a_{13} \ln (E D U)_{i, t}+a_{14} \ln (P O L I)_{i, t}+\epsilon_{i, t}
\end{gathered}
$$

$$
\begin{gathered}
\ln (L P I 6)_{i, t}=a_{0}+a_{1} \ln (F D I)_{i, t}+a_{2} \ln (G D P P C)_{i, t}+a_{3} \ln (T O P)_{i, t}+ \\
a_{4} \ln (I N D V)_{i, t}+a_{5} \ln (M V D)_{i, t}+a_{6} \ln (F O S S I L)_{i, t}+a_{7} \ln (T G H G)_{i, t}+ \\
a_{8} \ln (N O X)_{i, t}+a_{9} \ln (C O 2)_{i, t}+a_{10} \ln (R E C)_{i, t}+a_{11} \ln (\mathrm{HXP})_{i, t}+ \\
a_{12} \ln (\text { CRIME })_{i, t}+a_{13} \ln (E D U)_{i, t}+a_{14} \ln (P O L I)_{i, t}+\epsilon_{i, t}
\end{gathered}
$$

Where, LPI1 represents logistics performance index i.e., competitive priced shipment, LPI2 shows customs clearance process, LPI3 shows consignment tracking, LPI4 represents logistics quality services, LPI5 indicates transport and trade infrastructure, and LPI6 represents consignment schedule to reach within expected time. Envt 
represents environmental indicator covering FFUEL (fossil fuel energy consumption), $\mathrm{CO} 2$ (carbon emissions), REC (renewable energy consumptions), TGHT (total greenhouse gas emissions and nitrous oxide emissions. Soci shows social factors including HXP (health spending as percent of GDP), POLI (countries' political stability index), CRIME (crime rate proxy by intentional homicides rate), and EDU (government expenditure on education total \% of GDP) and ECO illustrates economic growth factors such as GDPPC (gross domestic product on per capita), MVD (manufacturing value added), TOP (trade openness), FDI (foreign direct investment inflows), and IVD (industry value added), while the constant and error correction in the model are represented as $a$ and $\in$ respectively.

This research employs FMOLS and DOLS for testing hypothesis. Before we go to parameter estimates for the above six equations, four different panel unit root tests are used, including Im et al. (2003) Levin et al. (2002) PP and Fisher Chi-square and ADF tests. After the satisfactory results of panel unit root test, this research evaluates the long-term cointegration among endogenous and exogenous variables. Finally, the research uses non-parametric panel FMOLS and parametric technique of DOLS estimators for robustness. Pedroni (2001) and Pedroni (1999) built a sing-equation technique to calculate cointegration vector for panel data set including DOLS and FMOLS. They both corrected the problem of serial correlation and endogenity from the model (Pedroni 2000).

\section{Results and Discussion}

Table 1 shows the descriptive statistics and most exogenous and endogenous variables have a positive mean and standard deviation, which indicates high logistics performance including LPI 1 (competitive priced shipment), LPI 2 (customs clearance process), LPI 3,(consignment tracking) LPI 4,(logistics quality services) LPI5 (transport and trade infrastructure), and LPI6 (consignment schedule to reach within expected time). The positive and great value of logistics performance contributes to sustainable economic development and growth, improving FDI net inflows (\% of GDP), MVD (manufacturing value added annual \% growth), TOP (trade openness \% of GDP), IVD (industry value added annual \% growth).

Table 1: The descriptive statistics

\begin{tabular}{|lcc|}
\hline Variables & Mean & Std. Dev. \\
\hline LPI1 & 2.158 & 0.4112 \\
LPI2 & 2.279 & 0.4555 \\
LPI3 & 2.521 & 0.4145 \\
LPI4 & 2.423 & 0.4241 \\
LPI5 & 2.621 & 0.4725 \\
LPI6 & 2.647 & 0.4612 \\
FDI & 19.51479 & 8.9314 \\
GDPPC & 21.49234 & 10551 \\
TOP & 13.11547 & 2.113 \\
\end{tabular}




\begin{tabular}{|lcc|} 
INDV & 2.018922 & 2.779 \\
MVD & 61.01469 & 37.572 \\
FOSSIL & 9.821401 & 5.072 \\
TGHG & 498413 & 717409 \\
NOX & 0.908142 & 6102468 \\
CO2 & 0.8048382 & 0.6701458 \\
REC & 51.85719 & 26.27372 \\
HXP & 9.125881 & 2.308145 \\
POLI & -1.089514 & 1.117581 \\
CRIME & 11.584 & 0.6571 \\
EDU & 7.2578 & 0.2257 \\
\hline
\end{tabular}

Note: *LPI1 indicates logistics performance index i.e., competitive priced shipment, LPI2 indicates customs clearance process, LPI3 shows consignment tracking, LPI4 represents logistics quality services, LPI5 indicates transport and trade infrastructure, and LPI6 represents consignment schedule to reach within expected time

While, environmental beauty and HXP (health expenditure spending \% of GDP) are influenced by greater $\mathrm{CO} 2$ (carbon emissions) in metric tons per capita, FFUEL (fossil fuel energy consumption) in total energy consumption, NOX (nitrogen emissions) thousand metric tons of $\mathrm{CO} 2$ equivalent, TGHG (total greenhouse gas emissions) in metric tons per capita, and they can be reduced through greater EDU (education \% of GDP) and utilization of REC (renewable energy consumption) in logistics operations. There is no doubt that POLI (countries political stability) improves logistics service and builds new logistics infrastructure with greater trade and business opportunities. But regrettably, in most Asian emerging economies, POLI has negative mean and standard deviation, which directly and indirectly disturbs the economic, social, and environmental sustainability in terms of ineffective and/or poor policies, unpredictable trend in stock markets, and delays in governmental projects due to political interference.

Table 2 displays the correlation. It can be seen that there are different impacts of logistics operations on economic, social and environmental indicators, as logistics operations have a positive impact on economic indicators including, TOP (trade openness), GDP (\% of per capita income), FDI (foreign direct investment inflows), IVD (industry value added) and MVD (manufacturing value added), while logistics operations are also a significant contributor to CO2 (carbon emissions), NOX (nitrogen emissions), and TGHG (greenhouse gas emissions) due to greater level of FOSSIL (fossil fuel consumptions), and they can be controlled/mitigated through increasing EDU (education \% of GDP) and adopting RE (renewable energy) as an substitute of fossil fuel and energy. Political stability creates numerous business and trade opportunities through effective and improved polices and good relationship with other countries. However, most Asian emerging economies are suffering terrorism and poor political stability, negatively influencing economic growth and reflecting negative association with GDPPC, IVD, FDI, TOP, and logistics operations. 
Table 2: Correlation Matrix

\begin{tabular}{|c|c|c|c|c|c|c|c|c|c|c|c|c|c|c|c|c|c|c|c|c|}
\hline Variables & LPI1 & LPI2 & LPI3 & LPI4 & LPI5 & LPI6 & FDI & GDPPC & TOP & INDV & MVD & FOSSIL & TGHG & NOX & $\mathrm{CO} 2$ & REC & HXP & CRIME & EDU & POLI \\
\hline LPI1 & 1 & & & & & & & & & & & & & & & & & & & \\
\hline LPI2 & 0.960 & 1 & & & & & & & & & & & & & & & & & & \\
\hline LPI3 & 0.900 & 0.901 & 1 & & & & & & & & & & & & & & & & & \\
\hline LPI4 & 0.800 & 0.852 & 0.707 & 1 & & & & & & & & & & & & & & & & \\
\hline LPI5 & 0.752 & 0.819 & $0 . .798$ & 0.991 & 1 & & & & & & & & & & & & & & & \\
\hline LPI6 & 0.795 & 0.798 & 0.721 & 0.985 & 0.779 & 1 & & & & & & & & & & & & & & \\
\hline FDI & 0.598 & 0.985 & 0.661 & 0.779 & 0.985 & 0.611 & 1 & & & & & & & & & & & & & \\
\hline GDPPC & 0.874 & 0.115 & 0.114 & 0.128 & 0.554 & 0.781 & 0.655 & 1 & & & & & & & & & & & & \\
\hline TOP & 0.745 & 0.254 & 0.357 & 0.451 & 0.257 & 0.254 & 0.635 & 778 & 1 & & & & & & & & & & & \\
\hline INDV & 0.109 & 0.365 & 0.421 & 0.526 & 0.776 & 0.652 & 0.471 & 0.547 & 0.887 & 1 & & & & & & & & & & \\
\hline MVD & 0.118 & 0.887 & 0.789 & 0.589 & 0.713 & 0.736 & 0.951 & 0.921 & 0.571 & 0.775 & 1 & & & & & & & & & \\
\hline FOSSIL & 0.874 & 0.257 & 0.289 & 0.715 & 0.924 & 0.895 & 0.877 & 0.716 & 0.651 & 0.798 & 0.773 & 1 & & & & & & & & \\
\hline TGHG & 0.191 & 0.981 & 0.125 & 0.742 & 0.824 & 0.798 & 0.456 & 0.755 & 0.889 & 0.826 & 0.871 & 0.718 & 1 & & & & & & & \\
\hline NOX & 0.254 & 0.119 & 0.223 & 0.941 & 0.913 & 0.936 & 0.718 & 0.697 & 0.715 & 0.651 & 0.776 & 0.712 & 0.981 & 1 & & & & & & \\
\hline $\mathrm{CO} 2$ & 0.125 & 0.325 & 0.784 & 0.715 & 0.781 & 0.791 & 0.896 & 0.997 & 0.689 & 0.789 & 0.988 & 0.548 & 0.872 & 0.741 & 1 & & & & & \\
\hline REC & -0.658 & -0.784 & -0.887 & -0.654 & -0.814 & -0.884 & 0.476 & 0.819 & 0.871 & 0.556 & 0.711 & -0.873 & -0.882 & -0.651 & -0.871 & 1 & & & & \\
\hline HXP & 0.771 & 0.796 & 0.776 & 0.717 & 0.911 & 0.895 & 0.784 & 0.888 & 0.778 & 0.914 & 0.893 & 0.917 & 0.754 & 0.897 & 0.715 & 0.889 & 1 & & & \\
\hline CRIME & 0.895 & 0.854 & 0.811 & 0.987 & 0.877 & 0.716 & 0.911 & 0.799 & 0.689 & 0.828 & 0.654 & 0.661 & 0.897 & 0.887 & 0.570 & 0.547 & 0.871 & 1 & & \\
\hline EDU & 0.213 & 0.254 & 0.221 & 0.354 & 0.229 & 0.341 & 0.247 & 0.411 & 0.571 & 0.871 & 0.732 & -0.547 & -0.658 & -0.619 & -0.694 & 0.658 & 0.446 & 0.547 & 1 & \\
\hline POLI & -0.789 & -0.985 & 0.227 & -0.229 & -0.313 & -0.651 & 0.288 & -0.411 & -0.354 & -0.786 & 0.812 & 0.571 & 0.469 & 0.789 & 0.845 & 0.547 & 0.584 & 0.715 & 0.814 & 1 \\
\hline
\end{tabular}

Note: *LPII indicates logistics performance index i.e., competitive priced shipment, LPI2 indicates customs clearance process, LPI3 shows consignment tracking, LPI4 represents logistics quality services, LPI5 indicates transport and trade infrastructure, and LPI6 represents consignment schedule to reach within expected time.

* All bold values show significant associations. 
Table 3 demonstrates the panel unit root test and shows that, there is a mixture order of integration among the variables' series. On the other hand, it is found that these variables are difference stationary in at least two of the suggested panel unit root tests. Thus, we draw the conclusion that the variables hold I(1) series.

Table 3: Panel unit root - Summary

\begin{tabular}{|c|c|c|c|c|c|c|c|c|}
\hline \multirow[b]{2}{*}{ Variables } & \multicolumn{2}{|c|}{ IPS } & \multicolumn{2}{|c|}{ LLC } & \multicolumn{2}{|c|}{$\mathbf{P P}$} & \multicolumn{2}{|c|}{ ADF } \\
\hline & Level & $\begin{array}{l}\text { First } \\
\text { Difference }\end{array}$ & Level & $\begin{array}{l}\text { First } \\
\text { Difference }\end{array}$ & Level & $\begin{array}{l}\text { First } \\
\text { Difference }\end{array}$ & Level & $\begin{array}{l}\text { First } \\
\text { Difference }\end{array}$ \\
\hline FDI & -0.197 & $-3.061^{*}$ & -0.195 & $-3.061^{*}$ & $25.15^{*}$ & $55.621^{*}$ & 13.11 & $22.654^{* *}$ \\
\hline GDPPC & 0.294 & 0.745 & $1.397^{* * *}$ & 0.035 & 4.559 & $17.961 * * *$ & 6.412 & 8.521 \\
\hline TOP & -0.323 & $-2.114 * *$ & 0.811 & $-2.772 * *$ & 7.041 & $47.651^{*}$ & 15.12 & $20.125^{* *}$ \\
\hline INDV & -0.561 & $-3.045^{* *}$ & 0.557 & $-3.441^{*}$ & 5.125 & $35.110^{*}$ & 12.214 & $18.654^{*}$ \\
\hline MVD & -0.341 & $-3.564^{*}$ & -0.448 & $-2.542 *$ & 4.544 & $65.311^{*}$ & 11.544 & 19.254 \\
\hline FOSSIL & 1.823 & $-1.496^{*}$ & 1.494 & $-2.291^{*}$ & 3.553 & $51.806^{*}$ & 2.007 & $15.291 *$ \\
\hline TGHG & -0.125 & $-2.254^{*}$ & -0.525 & $-3.221^{*}$ & 3.544 & $71.254^{*}$ & 9.254 & $11.214^{*}$ \\
\hline NOX & 0.154 & -3.541 & -0.254 & $-3.145^{*}$ & 4.254 & $51.454^{*}$ & 18.214 & 43.652 \\
\hline $\mathrm{CO} 2$ & -1.021 & $-1.733^{* *}$ & -1.021 & $-2.691^{*}$ & $16.86^{* * *}$ & $41.738^{*}$ & 14.921 & $19.530 *$ \\
\hline REC & -0.223 & $-2.654^{*}$ & -0.321 & $-4.124 *$ & 4.254 & $45.514^{*}$ & 19.521 & $46.214 *$ \\
\hline HXP & -0.546 & $-1.256^{*}$ & -0.314 & $-3.440 * *$ & 3.685 & $73.541^{*}$ & 9.541 & $16.214 *$ \\
\hline CRIME & 1.039 & $-1.655^{* *}$ & -0.714 & $-2.124 * *$ & 3.214 & $78.151^{*}$ & 18.71 & $42.211^{* *}$ \\
\hline EDU & -0.324 & $-1.789^{*}$ & 0.785 & $-3.897^{*}$ & 4.215 & $63.214^{*}$ & 15.241 & $23.214^{* *}$ \\
\hline POLI & -2.902 & -6.078 & -0.118 & $-3.181^{*}$ & 5.322 & $55.361^{*}$ & 29.494 & 42.439 \\
\hline LPI1 & $2.275^{*}$ & $-4.207^{*}$ & $-0.112^{*}$ & $-3.181^{*}$ & 4.314 & $69.651^{*}$ & 6.584 & $36.049 *$ \\
\hline LPI2 & -4.798 & $-9.196^{*}$ & $1.681^{*}$ & $-3.180 *$ & 6.652 & $74.839^{*}$ & $25.421^{*}$ & $59.482 *$ \\
\hline LPI3 & $-2.559^{*}$ & $-8.043^{*}$ & $-4.616^{*}$ & $-11.681^{*}$ & 5.291 & $75.421^{*}$ & $20.352 *$ & $47.413^{*}$ \\
\hline LPI4 & -2.175 & $-5.784^{*}$ & $-3.155^{*}$ & $-7.684^{*}$ & 9.776 & $72.858^{*}$ & $18.52^{*}$ & $41.265^{*}$ \\
\hline LPI5 & -0.098 & $-5.781 *$ & $-3.735^{*}$ & $-4.781^{*}$ & 3.682 & $72.017^{*}$ & $13.35^{* *}$ & $20.350 *$ \\
\hline LPI6 & $0.325^{* *}$ & -2.118 & -0.171 & $-2.771^{*}$ & 7.044 & $47.659^{*}$ & $18.45^{* *}$ & $42.545^{*}$ \\
\hline
\end{tabular}

Note: LPI1 indicates logistics performance index i.e., competitive priced shipment, LPI2 indicates customs clearance process, LPI3 shows consignment tracking, LPI4 represents logistics quality services, LPI5 indicates transport and trade infrastructure, and LPI6 represents consignment schedule to reach within expected time.

$*, * *$, and $* * *$ shows $10 \%, 5 \%$, and $1 \%$ level of significance.

Trend Assumption: Individual trend and individual intercept. 
Table 4 shows the panel cointegration estimates by pedroni's non-parametric approach and indicates that PP-statistics, Group ADF and Panel-statistics are significant at least $10 \%$ level. Therefore it confirmed the rejection of no cointegration in all 6 models.

Table 4: Panel cointegration results

\begin{tabular}{lrrrrrr}
\hline Panel Statistics & LPI 1 & LPI 2 & LPI 3 & LPI 4 & LPI 5 & LPI 6 \\
\hline \hline Panel v-Statistic & -1.021 & -0.687 & 0.104 & -1.011 & -0.573 & -0.994 \\
Panel rho-Statistic & 3.373 & 2.486 & 2.338 & 2.736 & 2.665 & 2.632 \\
Panel PP-Statistic & $-3.502^{*}$ & $-3.617^{*}$ & $-2.7338^{*}$ & $-4.289^{*}$ & $-3.428^{*}$ & $-4.487^{*}$ \\
Panel ADF-Statistic & -3.608 & $-1.389^{* * *}$ & $-0.519^{*}$ & $-1.358^{* * *}$ & $-0.887^{* *}$ & -1.718 \\
Group rho-Statistic & 3.353 & 2.389 & 2.635 & 2.589 & 2.841 & 2.479 \\
Group PP-Statistic & $-17.484^{*}$ & $-7.906^{*}$ & $-4.674^{*}$ & $-7.652^{*}$ & $-8.986^{*}$ & $-8.458^{*}$ \\
Group ADF-Statistic & -2.886 & -9.461 & $0.127^{*}$ & 0.130 & -0.842 & -0.836 \\
\hline
\end{tabular}

Note: LPI1 indicates logistics performance index i.e., competitive priced shipment, LPI2 indicates customs clearance process, LPI3 shows consignment tracking, LPI4 represents logistics quality services, LPI5 indicates transport and trade infrastructure, and LPI6 represents consignment schedule to reach within expected time.

$*$, *, and *** shows $10 \%, 5 \%$, and $1 \%$ level of significance. 
Table 5: FMOLS and DOLS estimations

\begin{tabular}{|c|c|c|c|c|c|c|c|c|c|c|c|c|}
\hline \multirow[t]{2}{*}{ Variables } & \multicolumn{6}{|c|}{ Panel FMOLS Estimator } & \multicolumn{6}{|c|}{ Panel DOLS Estimator } \\
\hline & $\ln ($ LPI-1) & $\ln ($ LPI-2) & $\ln ($ LPI-3) & $\ln ($ LPI-4) & $\ln ($ LPI-5) & $\ln ($ LPI-6) & $\ln ($ LPI-1) & $\ln (\mathrm{LPI}-2)$ & $\ln (\mathrm{LPI}-3)$ & $\ln (\mathrm{LPI}-4)$ & $\ln ($ LPI-5) & $\ln ($ LPI-6) \\
\hline FDI & 0.001 & -0.001 & 0.003 & 0.006 & -0.0009 & 0.007 & -0.007 & -0.011 & 0.003 & 0.001 & -0.008 & -0.002 \\
\hline GDPPC & 0.062 & $0.082 * *$ & 0.179 & $0.002 * *$ & $0.062 * * *$ & 0.025 & 0.034 & $0.054^{*}$ & 0.154 & $0.024 * *$ & $0.048 * * *$ & -0.006 \\
\hline TOP & -0.013 & 0.043 & 0.017 & $0.003 * *$ & $0.029^{* *}$ & 0.027 & 0.002 & 0.028 & -0.051 & -0.014 & $0.055^{*}$ & 0.021 \\
\hline INDV & $0.045^{*}$ & $0.048^{*}$ & 0.084 & $0.041^{*}$ & $0.038^{* *}$ & 0.087 & 0.029 & $0.019 *$ & 0.055 & 0.028 & $0.063 *$ & $0.071^{*}$ \\
\hline MVD & 0.052 & $0.051^{* *}$ & 0.057 & $0.061^{* *}$ & 0.068 & 0.072 & 0.044 & $0.038^{*}$ & $0.045^{*}$ & $0.018^{*}$ & 0.058 & 0.050 \\
\hline FOSSIL & 0.355 & $0.071^{* *}$ & 6.647 & $0.579 * * *$ & 0.472 & $0.652 *$ & 0.374 & $0.084^{*}$ & -0.418 & $0.539^{* *}$ & 0.436 & 0.665 \\
\hline TGHG & 0.156 & 0.123 & 0.231 & 0.181 & 0.152 & 0.166 & 0.177 & 0.119 & 0.121 & 0.123 & 0.138 & 0.29 \\
\hline NOX & 0.131 & 0.157 & 0.137 & 0.133 & 0.149 & 0.150 & 0.161 & 0.167 & 0.178 & 0.177 & 0.183 & 0.187 \\
\hline $\mathrm{CO} 2$ & 0.124 & 0.010 & 0.026 & $0.085^{* *}$ & 0.125 & $0.187^{*}$ & 0.178 & 0.044 & 0.033 & $0.060^{*}$ & 0.173 & 0.72 \\
\hline REC & 0.021 & -0.029 & 0.016 & $0.011^{* * *}$ & -0.021 & 0.024 & -0.047 & 0.048 & 0.049 & $0.019^{* *}$ & -0.051 & $0.053^{*}$ \\
\hline HXP & $-0.081 *$ & $-0.091 *$ & -0.088 & $-0.073^{*}$ & $-0.069 * *$ & 0.082 & -0.087 & $-0.065^{*}$ & -0.047 & $-0.055^{* *}$ & $-0.075^{* *}$ & 0.079 \\
\hline CRIME & 0.097 & -0.005 & 0.078 & -0.024 & 0.058 & 0.058 & 0.131 & 0.002 & 0.080 & -0.026 & 0.003 & 0.090 \\
\hline EDU & 0.057 & $0.047^{*}$ & $0.023 *$ & $0.056^{* *}$ & 0.061 & 0.028 & 0.041 & $0.046^{*}$ & 0.017 & $0.021 *$ & 0.029 & 0.031 \\
\hline POLI & -0.017 & $-0.015^{*}$ & -0.011 & $-0.021^{*}$ & $0.027^{* * *}$ & -0.016 & -0.012 & -0.031 & -0.024 & $-0.033^{*}$ & $-0.022 * *$ & -0.018 \\
\hline \multicolumn{13}{|l|}{ Statistical Tests } \\
\hline R-squared & 0.944 & 0.985 & 0.878 & 0.964 & 0.948 & 0.812 & 0.974 & 0.992 & 0.895 & 0.982 & 0.972 & 0.918 \\
\hline Adj. R-squared & 0.941 & 0.982 & 0.875 & 0.958 & 0.943 & 0.791 & 0.971 & 0.991 & 0.884 & 0.981 & 0.971 & 0.912 \\
\hline S.E. of regression & 0.031 & 0.021 & 0.061 & 0.029 & 0.35 & 0.0451 & 0.021 & 0.016 & 0.059 & 0.017 & 0.025 & 0.029 \\
\hline Long-run variance & 0.001 & 0.0006 & 0.006 & 0.0007 & 0.001 & 0.001 & 0.0005 & 0.0003 & 0.005 & 0.0004 & 0.0008 & 0.0009 \\
\hline
\end{tabular}

Note: ${ }^{*} * *$, and $* * *$ indicates $10 \%, 5 \%$, and $1 \%$ level of significance 
Table 5 shows the results of FMOLS and DOLS estimators and robust parameter estimates are obtained. Five environmental indicators have been used in this study, i.e., fossil fuel, $\mathrm{CO} 2$ emissions, renewable energy, total greenhouse gas emissions, and nitrogen emissions under the influence of logistics performance index. The findings reveal that carbon emissions are significantly and positively correlated with LPI4 (logistics quality service) and LPI5 (transport and trade-related infrastructure). 1\% increase in logistics operations, trade and related transport infrastructure will increase carbon emissions in the atmosphere by $0.085 \%$ and $0.087 \%$. Undeniably, logistics operations are purely based on transportation movement, and meanwhile, transportation is a nonnegligible contributor to carbon emissions. Khan et al., (2018) and Dekker et al., (2012) highlighted that transport industry is a main reason for climate change, global warming, air and water pollution. An empirical study conducted by Zaman \& Shamsuddin, (2017) identifies the determinants of environmental pollution and the scope of green logistics in the context of European developed countries. The results show that environmental sustainability is seriously compromised due to heavy industrialization. Amid all the thriving sectors, logistics is a significantly contributor to carbon emissions, nitrogen and greenhouse gas emissions, which not only destroying the natural beauty of the planet but also poisoning to the agricultural lands, foods and vegetables. Khan et al., (2018) and Sharma \& Gandhi, (2016) research explored that logistics and transport sector is also a cause of several pollution intensive diseases such as lungs function weakening, pulmonary cancer, asthma attacks, and birth defects. Bechtsis et al., (2017) argued that heavy financial penalties should be imposed on polluted logistical operations and vehicles for better environmental sustainability. Bektas et al. (2016) and Khan et al., (2017a) emphasized the need of green practices and utilization of renewable energy in logistics and supply chain operations to controlled/mitigate harmful effects on environment and society.

The results indicates that fossil fuel is significantly and positively correlated with LP2 (customs clearance process), LPI4 (logistics quality services) and LPI6 (consignment schedule to reach within expected time) on $5 \%, 1 \%$ and $10 \%$ level of confidence, while $1 \%$ increase in LPI2, LPI4 and LPI6 will increase the fossil fuel consumption by $0.071 \%, 0.079 \%$ and $0.052 \%$, which heavily polluted natural beauty of environment and also a cause of several skin-related diseases due to acid rainfall and elimination of ozone layer. Zawaydeh, (2017) logistics sector heavily consume energy and fossil fuel, which emits carbon emissions and greenhouse gas emissions. Leigh \& Li, (2015) suggested in their research' recommendations that promoting biofuels and green energy is a better substitute of fossil fuel to mitigate problem of climate change and environmental degradation. Gold \& Seuring, (2011) highlighted that biofuels and renewable energy sources should be used in manufacturing and logistics industries to protect environmental sustainability, because these two industries playing significant role to polluting atmosphere. The adoption of clean, alternative sources of energy, like advanced biofuels and natural gas, is the first step to proceed green logistics implementation, while corporate sector itself cannot adopt green energy in their 
manufacturing and logistics operations without political support of environmentalfriendly legislation (Khan and Dong, 2017b; Abid et al., 2012). Aldakhil, (2018) conducted a panel study in the perspective of BRICS member countries to investigate the determinants of green logistics. They found that poor logistics and trade-related infrastructure increase maintenance cost of vehicles and fuel consumption, which is translated into air and water pollution.

However, REC (renewable energy consumption) has a positive relationship with LPI 4 (quality of logistics services) and 1\% increase renewable energy consumption will improve quality of logistics services by $0.011 \%$ on $1 \%$ level of confidence. So it is concluded that, renewable energy not only plays a vital role in greening environment but also plays a positive part in improving logistics operations. In fact, Bozan, (2015) confirmed that it is the most feasible to apply renewable energy to control environmental pollution without compromising on economic growth. Khan et al., (2018) highlighted that biofuel is cheaper than fossil fuel and it also maintains environmental resources. Mafakheri \& Nasiri, (2014) argued that green energy sources have narrow market so that they cannot be extended/broader without the support of regulatory authority in terms of pro-environmental legislation, subsidies and tax exemption on renewable energy projects (Khan et al., (2016a). Transportation and logistics industry is extensively based on fossil fuel, primarily causing global warming, air and water pollution, acid rainfall, poisoning sea food and agricultural land. Li, (2014) emphasized the usage of renewable energy in logistical operations for better environmental sustainability. Furthermore, enterprises may build their competitive edge through adoption of green practices and usage of renewable energy in their logistical operations such as improved firms' image and reputation, customer trust, and greater export opportunities to developed Western and European economies due to catering proenvironmental policies.

This panel study uses four economic growth and development factors including GDPPC (gross domestic product per capita), MVD (manufacturing value added), FDI (foreign direct investment inflows), TOP (trade openness), and IVD (industry value added) under the influence of logistics operations. Table 5 displays that GDPPC is positively and strongly correlated with LPI 2 (efficiency of customs clearance process), LPI 4 (quality of logistics services), and LPI 5 (trade and transport-related infrastructure) on $5 \%, 5 \%$, and $1 \%$ level of confidence respectively. The results show that $1 \%$ increase in LPI2, LPi4, and LPI5 will uplift gross domestic product per capita by $0.082 \%$, $0.002 \%$, and $0.062 \%$ respectively. Similarly, Zhang et al., (2018) carried out a study in the context of developed countries to explore the linkage between green logistics and economic development/growth. The findings revealed that logistics industry acts as a backbone in improving and uplifting economic growth of countries. In addition, Khan et al., (2018) draw a conclusion that poor logistical, trade and transport related infrastructure slow down the economic growth. The larger volume of trade openness is a positive sign and a reflection of healthy economic growth. The findings also reveal that trade openness and logistics operations have a strong positive relationship, while 
TOP (trade openness) increases by $0.003 \%$ and $0.029 \%$ due to improvement in quality of logistics services and transport infrastructure, which ease cross boarder and in bound logistics operations. Bose and Pal (2012) and Benitez et al. (2015) found there is a strong and positive relationship between enterprise greater financial performance and logistics operations. Besides, implementation of ecological and green ideology in logistics operations has a positive effect on economic development, FDI inflows and trade (Yang et al. 2016). Zhu and Sarkis (2004) found that green practices adopted in transport and logistics operations not only reduce environmental degradation but also enhance a firm's financial performance. Hausman et al. (2013) concluded that logistics performance in terms of time, flow of goods and cost has a considerable impact on trade volume that ultimately correlated with per capita income. Khan \& Dong (2017a) conducted a panel study and found that higher performance of logistics has a significant relationship with greater trade volume and countries' per capita income.

Table 5 shows that MVD (manufacturing value added) and IVD (industry value added) is positively correlated with LPI1, LPI2, LPI4, and LPI5 on different levels of confidence interval. Simply speaking, efficiency of customs clearance, quality of logistics and transport infrastructure enhance manufacturing and industry value added activities. In Asian emerging economies, Poor transport infrastructure, inefficient customs clearance processes, and worst quality of logistics mechanism, reduced to the business and trade opportunities with Western and European developed countries and also portray negative image of Asian developing due to bribery culture, political instability, polluted vehicles, inefficient processes and poor logistics and transport infrastructure, (Bölük \& Mert, 2015). From the results, we can see that there is a significant and positive correlation between greater manufacturing value added, industry value added, per capita income, trade openness and improved green logistics performance, while the findings are also supported by earlier published researches including (Zhang et al., 2018; Aldakhil et al., 2018; Khan and Dong, 2017b; Wanzala \& Zhihong, 2016; Khan et al., 2016a; Yune et al., 2016; Bölük, \& Mert, (2015); Ruparathna \& Hewage, 2015; Simpson, 2012; Wandersee et al., 2012). They highlighted that logistics operations mainly depend on fossil fuel so that logistics operations become a primary cause of environmental degradation. In addition, inefficient customs clearance process and poor logistics infrastructure become a obstacle to economic growth and development. Undeniably, in a number of Asian developing countries, customs clearance process is not so efficient as in Western world; their logistics and transport-related infrastructure is very old and broken in different patches due to several reasons including, non-seriousness of regulatory bodies, poor political stability, low foreign and domestic investments and high rate of corruptions, Furthermore, Asian developing nations are suffered from terrorism the most, which also negatively influences logistics industry mechanism and economic growth.

The article used four social indicators, i.e., POLI (countries' political stability index), EDU (education expenditure spending \% of GDP), CRIME (crime rate proxy by international homicides rate), and HXP (health expenditure spending \% of GDP). The 
findings indicate that POLI is inversely correlated with logistics operations including LPI2 LPI4 and LPI5. The results indicate that $1 \%$ increase in long-term political instability in the panel of selected countries will decrease the quality of logistics and transport infrastructure, and efficiency of customs clearance process by $0.021 \%$, $0.027 \%$, and $0.015 \%$ respectively. Unfortunately, most Asian developing countries political stability is very difficulty to obtain and maintain. In most times the government is under military control due to terrorism. Many Asian developing countries such as Afghanistan, Pakistan, India, and Myanmar economy have been diffused and governments have lost their credibility and power to enforce their control law. There are still certain regions suffering from worsening already volatile law and order situation. (Bush et al., 2015; Rodríguez et al., 2014). Zhang et al., (2018) and Heldeweg et al., (2015) highlighted that long-term political stability is an important factor for reducing social concerns, improving economic development and enhancing ecological friendly legislation effectiveness (Khan \& Dong, 2017a ; Acquaye et al., 2015; Tengku, et al., 2011). Since last couple of decades, most Asian countries have been suffering from different social, economic and environmental issues including, environmental deterioration, natural disaster, and military dictatorship, war with neighbor countries and anarchy situation, leading to poor economic, social and environmental sustainability.

The results indicate that EDU (government expenditure on education total \% of GDP) have positive relationship with logistics operations including, quality of logistics services, consignment tracking, and efficiency of customs clearance process, while $1 \%$ increase in education expenditure will obtain $0.056 \%, 0.023 \%$, and $0.047 \%$ increase in quality of logistics, consignment tracking and efficiency of customs clearance process respectively. Or more simply, education factor has a significant positive effect on logistics operations. Khan et al., (2016c) and Beitzen-Heineke et al., (2017) highlighted that greater level of relevant knowledge and training increase efficiency of labor force, which can be translated into better financial performance. Khan et al., (2016) discussed integration field theory and argued that manufacturing industry efficiency and performance are better than logistics industry, because mostly logistics firms' managerial staff are not competent enough. Zhang et al., (2018) and Beitzen-Heineke et al., (2017) environmental awareness training and programs enhance the customers, employees, and supply chain members' understanding on environmental problems. While, the results indicates that poor logistics services and trade-related infrastructure has negative relationship with HXP (health expenditure) on $1 \%$ and 5\% level of confidence. Simply speaking, $1 \%$ increase in the poor logistics and infrastructure is related with transport will increase health expenditure by $0.073 \%$ and $0.069 \%$ respectively. In most Asian emerging countries, logistics and transport-related infrastructure is in poor condition, and most bridges are very risky for heavy vehicles due to no maintenance from years ago. Furthermore, due to poor transport-related infrastructure, polluted vehicles and usage of fossil fuel in logistics operations, several pollution-intensive diseases are occurring such as high blood pressure, lungs problems, asthma attacks, and mental stress. Burrell et al., (2006) polluted operations of corporate 
sector have negative effects on our environment and even human health, due to water and air pollution. Khan et al., (2017a) concluded that people health has been compromised due to unsustainable industrialization. Mariano et al., (2017) found that logistics operations is primarily responsible for climate change, global warming and air pollution, and confirmed that its volatility to attain 'green' agenda that need to be controlled by economic policies to mitigate carbon emissions across the globe through usage of renewable energy and green practices in logistical operations. In addition, Maddala and Lahiri, (2006); Tsoulfas and Pappis, (2006) suggest that governmental bodies should tackle the situation and realize their responsibilities to enforce strict environmental-friendly legislation, encourage biofuels and green energy in logistical operations, and impose huge financial and social penalties on polluted manufacturing and/or logistics firms.

\section{Conclusion and policymaking}

Since last couple of decades, most developing countries in Asia have been suffering from terrorism, natural disasters, political instability, business lockdown strikes, and worst law and order situation particularly Afghanistan, India, Myanmar, Nepal, and Bhutan. They lost billions of dollars from their economy but also portray themselves negatively image on international media. In addition, these countries are highly affected by global warming and climate change due to ineffective environmental policies. Further, logistics sector is unable to fully contribute to economic growth due to poor logistical, trade and transport infrastructure. This debate is further extended with the "green corridor/practices and renewable energy". This panel study is conducted to investigate the correlation between green logistics indicators (LPI1 (competitive priced shipment), LPI2 (customs clearance process), LPI3 (consignment tracking), LPI4 (logistics quality services), LPI5 (transport and trade-related infrastructure), and LPI6 (consignment schedule to reach within expected time), social (crime rate, political instability, education \% of GDP and health expenditure \% of GDP), environmental (carbon emissions, greenhouse gas emissions, nitrogen emissions, and fossil fuel consumption) and economic factors (trade openness, foreign direct investment inflows, per capita income, manufacturing value added and industry value added) in Asian emerging economies.

The results indicate that logistics operations improve economic growth in terms of per capita income, trade openness and industry value added activities, while logistics operation is negatively correlated with environmental sustainability due to carbon emissions, greenhouse gas emissions, fossil fuel and nitrogen emissions; logistics operation is a cause of several pollution related with diseases such as ischaemic heart disease, stroke, lungs cancer, and acute lower respiratory infections in children, while these social and environmental problems can be mitigated through adoption of renewable energy and green practices in logistical operation without compromising economic growth. However, the findings revealed that most Asian developing countries have poor logistics, trade and transport infrastructure, in fact, most highways/bridges 
are very old and not suitable for traffic. In addition, since last couple of decades, Asian region particularly developing countries have been suffering from different natural disaster, terrorist attacks and political instability, dragging down economic development and also negatively influencing social and environment performance of countries in terms of lack behind environmental-friendly policies.

This study suggests that governmental bodies and corporate sector should stand up to take their responsibility for protecting social and environmental sustainability. First, government should provide subsidies on green products and tax exemptions on green projects and green vehicles to encourage green ideology. Second, regulatory authorities need to build environmental-friendly policies for protection natural resources and beauty. Third, corporate sector should use renewable energy in their logistical and manufacturing operations as a substitute of fossil fuel, which significantly reduces carbon emissions in the atmosphere. Fourth, government may restrict the movement of heavy transport in night time, which will not create congestion on roads but also reduce consumption of fossil fuels.

\section{References}

Khan, S.A.R., Yu, Z., Anees, M., Golpîra, H., Lahmar, A., Dong Q. (2018). Green Supply Chain Management, Economic Growth and Environment: A GMM Based Evidence. Journal of Cleaner Production, 185(6), 588-599.

Aldakhil, A. M., Nassani, A. A., Awan, U., Abro, M. M. Q., Zaman, K. (2018). Determinants of green logistics in BRICS countries: An integrated supply chain model for green business. Journal of Cleaner Production, 195, pp. 861-686. https://doi.org/10.1016/j.jclepro.2018.05.248.

Khan, S.A.R., and Dong, Q. (2017a). Does national scale economic and environmental indicators spur logistics performance? Evidence from UK. Environmental Science and Pollution Research, 24(34), 26692-26705.

Martel, A., \& Klibi, W. (2016). Supply Chains: Issues and Opportunities. In Designing Value Creating Supply Chain Networks (pp. 1-43). Springer International Publishing.

Khan, S.A.R., Dong, Q. (2017b). Impact of green supply chain management practices on firms' performance: an empirical study from the perspective of Pakistan. Environmental Science and Pollution Research, 24(20), 16829-16844.

Khan, S.A.R., Dong, Q., Yu, Z. (2016a). Research on the Measuring Performance of Green Supply Chain Management: In the Perspective of China. Journal of Engineering Research in Africa, 27, 167-178.

Zaman, K., \& Shamsuddin, S. (2017). Green logistics and national scale economic indicators: Evidence from a panel of selected European countries. Journal of Cleaner Production, 143, 51-63. https://doi.org/10.1016/j.jclepro.2016.12.150

Petrini, M., \& Pozzebon, M. (2009). Managing sustainability with the support of business intelligence: integrating socio-environemtnal indicators and organisational context. The Journal of Strategic information Systems, 18(4), 178-191.

Lai, K. H., Ngai, E, W. T., \& Cheng, T.C.E. (2004). An empirical study of supply chain performance in transport logistics, International Journal of Production Economics, 87(3), 321-331. 
Nikolaou, I. E., Evangelinos, K. I., \& Allan, S. (2013). A reserve logistics social responsibility evaluation framework based on the triple bottom line approach., Journal of Cleaner Production, 56, 173-184.

Ruamsook, K., Russell, D. M., \& Thomchick, E. A. (2009). Sourcing from low-cost countries: Identifying sourcing issues and prioritizing impacts on logistics performance. The International Journal of Logistics Management, 20(1), 79-96.

Acquaye, A., Feng, K., Oppon, E., Salhi, S., Ibn-Mohammed, T., Genovese, A., \& Hubacek, K. (2017). Measuring the environmental sustainability performance of global supply chains: A multi-regional input-output analysis for carbon, sulphur oxide and water footprints, Journal of environmental management, 187, 571-585.

Acquaye, A., Genovese, A., Barrett, J., \& Lenny Koh, S. C. (2014). Benchmarking carbon emissions performance in supply chains. Supply chain Management: An International Journal, 19(3), 306321.

Acquaye, A. A., Yamoah, F. A., \& Feng, K. (2015). An integrated environmental and fairtrade labelling scheme for product supply chains. International Journal of Production Economics, 164, 472-483. https://doi.org/10.1016/j.ijpe.2014.12.014.

Khan, S.A.R., Zaman, K., Zhang, Y. (2016b). The relationship between energy-resource depletion, climate change, health resources and the environmental Kuznets curve: Evidence from the panel of selected developed countries. Renewable and Sustainable Energy Reviews, 62, 468-477. DOI: 10.1016/j.rser.2016.04.061.

Sarkis, J., Helms, M. M., \& Hervani, A. A. (2010). Reverse logistics and social sustainability, Corporate social responsibility and environmental management, 17(6), 337-354.

Van Hoek, R. I. (1999). From reversed logistics to green supply chains, Supply chain management: An international Journal, 4(3), 129-135.

Chaudhry, N., Roubaud, D., Akhter, W., Shahbaz M. (2018) impact of terrorism on stock markets: empirical evidence from the SAARC region, Finance Research Letters, , DOI:

10.1016/j.frl.2018.02.024.

Khan, S.A.R., Dong, Q., SongBo, W., Zaman, K., Zhang, Y. (2017a). Environmental logistics performance indicators affecting per capita income and sectoral growth: evidence from a panel of selected global ranked logistics countries. Environmental Science and Pollution Research, 24(2), 1518-1531.

Bektas, Y., Rodriguez-Salus, M., Schroeder, M., Gomez, A., Kaloshian, I., \& Eulgem, T. (2016). The Synthetic Elicitor DPMP (2,4-dichloro-6- $\{(\mathrm{E})-[(3-\mathrm{methoxyphenyl)imino]methyl}\}$ phenol) Triggers Strong Immunity in Arabidopsis thaliana and Tomato. Nature Scientific Reports, 6:29554 | DOI: 10.1038/srep2955,

Burrell, L., Hewlett, S. A., Luce, C. B., \& Frohman, D. (2006). K g Str gy out of His Mouth, (December).

Rodríguez, L. C., Montiel, I., \& Ozuna, T. (2014). A Conceptualization of How Firms Engage in Corporate Responsibility Based on Country Risk. https://doi.org/10.1177/0007650312475123

Khan, S.A.R., Dong, Q., and Zhang, Y. (2016c). Study of Logistics and Manufacturing Industry Integration from the Perspective of Pakistan, Journal of Engineering Research in Africa, DOI: 10.4028/www.scientific.net/JERA.24.172

Im, K. S., Pesaran, M. H., \& Shin, Y. (2003). Testing for unit roots in heterogeneous panels, Journal of Econometrics, 115, 53-74. 
Levin, A., Lin, C.F. \& Chu, J. (2002). Unit root in panel data: Asymptotic and finite-sample properties, Journal of Econometrics 108(1), 1-24.

Pedroni, P. (2001). Purchasing power party tests in cointegrated panels. Review of Economics and Statistics, 83, 723-741.

Pedroni, P. (2000). Fully modified OLS for heterogeneous cointegrated panels. Advances in Econometrics, 15, 93-130.

Pedroni, P. (1999). Fully modified OLS for heterogeneous cointegrated panels, Advances in Econometrica, 57, 1361-1401.

Pedroni, P. (1997). Panel cointegration, asymptotic and finite sample properties of pooled time series tests, with an application to the PPP hypothesis: new Results, Indiana University, Working Paper in Economics, November.

Dekker, R., Bloemhof, J., \& Mallidis, I. (2012). Operations Research for green logistics - An overview of aspects , issues , contributions and challenges. European Journal of Operational Research, 219(3), 671-679. https://doi.org/10.1016/j.ejor.2011.11.010

Sharma, S., \& Gandhi, M. A. (2016). Exploring Correlations in Components of Green Supply Chain Practices and Green Supply Chain Performance. Competitiveness Review: An International Business Journal, 26(3). https://doi.org/10.1108/CR-04-2015-0027

Bechtsis, D., Tsolakis, N., Vlachos, D., \& Iakovou, E. (2017). Sustainable supply chain management in the digitalisation era: The impact of Automated Guided Vehicles. Journal of Cleaner Production, 142, 3970-3984. https://doi.org/10.1016/j.jclepro.2016.10.057

Bektas, Y., Rodriguez-Salus, M., Schroeder, M., Gomez, A., Kaloshian, I., \& Eulgem, T. (2016). The Synthetic Elicitor DPMP (2,4-dichloro-6-\{(E)-[(3-methoxyphenyl)imino]methyl $\}$ phenol) Triggers Strong Immunity in Arabidopsis thaliana and Tomato. Nature Scientific Reports, 6:29554 | DOI: 10.1038/srep2955,

Zawaydeh, S. (2017). Economic, Environmental and Social Impacts of Developing Energy from Sustainable Resources in Jordan. Strategic Planning for Energy and the Environment, 36(3), 2452. https://doi.org/10.1080/10485236.2017.1181016p.

Leigh, M., \& Li, X. (2015). Industrial ecology, Bechtsis symbiosis and supply chain environmental sustainability : a case study of a large UK distributor. Journal of Cleaner Production, 106, 632643. https://doi.org/10.1016/j.jclepro.2014.09.022

Gold, S., \& Seuring, S. (2011). Supply chain and logistics issues of bio-energy production. Journal of Cleaner Production, 19(1), 32-42. https://doi.org/10.1016/j.jclepro.2010.08.009

Abid, M., Abdallah, K.B., \& Mraihi, R. (2012). Causality relationship between energy industrial consumption and economic growth : application on Tunisian country, Published in 2012 First International Conference on Renewable Energies and Vehicular Technology, 396-404. 10.1109/REVET.2012.6195303

Bozan, K. (2015). The impact of sustainable governance and practices on business performance : an empirical investigation of global firms, Pratim Datta Venugopal Gopalakrishna-Remani, 7(2), $97-120$.

Mafakheri, F., \& Nasiri, F. (2014). Modeling of biomass-to-energy supply chain operations: Applications, challenges and research directions. Energy Policy, 67, 116-126. https://doi.org/10.1016/j.enpol.2013.11.071

Li, Y. (2014). Environmental innovation practices and performance : moderating effect of resource commitment. Journal of Cleaner Production, 66, 450-458. 
https://doi.org/10.1016/j.jclepro.2013.11.044

Zhang, Y., Hêriş, G., and Khan, S.A.R. (2018) The relationship between green supply chain performance, energy demand, economic growth and environmental sustainability: An empirical evidence from developed countries, Log Forum-Scientific Journal of Logistics, 14(4), 479-498.

Bose, J., Pal, R. (2012). Do green supply chain management initiatives impact stock prices of firms?. Decision Support Systems, 52(3), 624-634.

Benitez, J., LIorens, J., Femandez, V. (2015). IT impact on talent management and operational environmental sustainability. Information Technology \& Management, 16(3), 207-220.

Yang, Z., Sun, J., Zhang, Y., Wang, Y. (2016). Peas and Carrots just because they are green? Operational fit between green supply chain management and green information system. Information Systems Frontiers, 20(3), 627-645. https://doi.org/10.1007/s10796-016-9698-y.

Zhu, Q., \& Sarkis, J. (2004). Relationships between operational practices and performance among early adopters of green supply chain management practices in Chinese manufacturing enterprises, Journal of Operations management, 22(3), 265-289.

Hausman, W. H., Lee, H. L., \& Subramanian, U. (2013). The impact of logistics performance on trade, Production and Operations Management, 22(2), 236-252.

Wanzala, W. G., \& Zhihong, J. (2016). Integration of the extended gateway concept in Supply Chain disruptions Management in East Africa-Conceptual paper. International Journal of Engineering Research in Africa, 20, 235-247. https://doi.org/10.4028/www.scientific.net/JERA.20.235

Yune, J. H., Tian, J., Liu, W., Chen, L., \& Descamps-Large, C. (2016). Greening Chinese chemical industrial park by implementing industrial ecology strategies: A case study. Resources, Conservation and Recycling, 112, 54-64. https://doi.org/10.1016/j.resconrec.2016.05.002

Bölük, G., \& Mert, M. (2015). The renewable energy, growth and environmental Kuznets curve in Turkey: An ARDL approach. Renewable and Sustainable Energy Reviews, 52, 587-595. https://doi.org/10.1016/j.rser.2015.07.138

Ruparathna, R., \& Hewage, K. (2015). Sustainable procurement in the Canadian construction industry: challenges and benefits. Canadian Journal of Civil Engineering, 42(6), 417-426. https://doi.org/10.1139/cjce-2014-0376

Simpson, D. (2012). Knowledge resources as a mediator of the relationship between recycling pressures and environmental performance. Journal of Cleaner Production, 22(1), 32-41. https://doi.org/10.1016/j.jclepro.2011.09.025.

Wandersee, S. M., An, L., López-Carr, D., \& Yang, Y. (2012). Perception and decisions in modeling coupled human and natural systems: A case study from Fanjingshan National Nature Reserve, China. Ecological Modelling, 229, 37-49. https://doi.org/10.1016/j.ecolmodel.2011.08.004

Bush, S. R., Oosterveer, P., Bailey, M., \& Mol, A. P. J. (2015). Sustainability governance of chains and networks: A review and future outlook. Journal of Cleaner Production, 107, 8-19. https://doi.org/10.1016/j.jclepro.2014.10.019.

Heldeweg, M. A., Sanders, M., \& Harmsen, M. (2015). Public-private or private-private energy partnerships? Toward good energy governance in regional and local green gas projects. Energy, Sustainability and Society, 5(9), 234-265. https://doi.org/10.1186/s13705-015-0038-8

TENGKU, H., TENGKU, ADELINE, ADURA. (2011). Making Sense of Environmental Governance: A Study of E-waste in Malaysia, Durham theses, Durham University. Available at Durham ETheses Online: http://etheses.dur.ac.uk/670/ [Accessed on November, 2017]

Beitzen-Heineke, E. F., Balta-Ozkan, N., \& Reefke, H. (2017). The prospects of zero-packaging 
grocery stores to improve the social and environmental impacts of the food supply chain. Journal of Cleaner Production, 140, 1528-1541. https://doi.org/10.1016/j.jclepro.2016.09.227

Mariano, E. B., Gobbo Jr, J. A., de Castro Camioto, F., \& do Nascimento Rebelatto, D. A. (2017). $\mathrm{Co} 2$ emissions and logistics performance: a composite index proposal, Journal of Cleaner Production, 163, 166-178.

Maddala, G.S., Lahiri, K. (2006). Introduction to Econometrics, fourth ed. Wiley, New York.

Tsoulfas, G.T., and Pappis, C.P. (2006). Environmental principles applicable to supply chains design and operation. Journal of Cleaner Production, 14(18), 1593-1602. 